\title{
On the existence of the global conformal gauge in string theory
}

\author{
M. O. Katanaev ${ }^{\mathrm{a}}$ \\ Steklov Mathematical Institute, ul. Gubkina, 8, Moscow 119991, Russia
}

Received: 30 April 2021 / Accepted: 30 June 2021 / Published online: 5 July 2021

(C) The Author(s) 2021

\begin{abstract}
The global conformal gauge is playing the crucial role in string theory providing the basis for quantization. Its existence for two-dimensional Lorentzian metric is known locally for a long time. We prove that if a Lorentzian metric is given on a plain then the conformal gauge exists globally on the whole $\mathbb{R}^{2}$. Moreover, we prove the existence of the conformal gauge globally on the whole worldsheets represented by infinite strips with straight boundaries for open and closed bosonic strings. The global existence of the conformal gauge on the whole plane is also proved for the positive definite Riemannian metric.
\end{abstract}

\section{Introduction}

The (super)string theory attracts much interest in physics and mathematics for the last fifty years (see, e.g., [1-3]). It is usually considered as the basis for construction of the unified quantum theory of all fundamental interactions including gravity. The crucial role in the theory is played by the conformal gauge for a metric of Lorentzian signature in which it is conformally flat. In fact, almost all results in string theory are obtained using the assumption that the conformal gauge exists on the whole string worldsheets represented by infinite strips with straight boundaries. For example, the covariant and light cone quantizations use Fourier series which exist only if the conformal gauge is applied on the whole string worldsheet.

The local existence of the conformal gauge is well known for a long time (see, for $\mathcal{C}^{2}$-metric, e.g., [4, Ch. I, $\S 6.1$ ], or [5, Ch. I, $\S 3.1])$. This gauge and boundary value problems were also considered in [6]. The local existence of the gauge is proved by writing down equations for transformation functions and considering their integrability conditions which guarantee the existence of solution in some neighbourhood of an arbitrary point. However it is not enough. In

a e-mail: katanaev@mi-ras.ru (corresponding author) string theory, it is assumed that the conformal gauge exists on an infinite strip with straight boundaries. There are two subtle questions: does the conformal gauge exist on the whole strip? and can the boundaries be made straight? In the present paper, we answer these questions affirmatively. The transition from local to global considerations is based on the global existence theorem for the solution of the Cauchy problem for two-dimensional hyperbolic differential equations with varying coefficients (see, e.g., [7], book IV, ch. I). This theorem is highly nontrivial, but allows one to make global statements.

The existence of the global conformal gauge, adopted in string theory, results in beautiful and consistent theory. Therefore the theory deserves attention by itself. But the question remains: are there other solutions of the initial Nambu-Goto string which are not captured by the usual approach? We prove that there are no such solutions. The main results of the present paper for Lorentzian signature metric are published in [8] without proofs.

The local existence of the conformal gauge (isothermal coordinates) for positive definite Riemannian metric is also known in mathematics for a long time. The proof for analytic metric is given e.g. in [4, Ch. I, § 6.4] and [5, Ch. I, § 3.4] and for $\mathcal{C}^{3}$-metric e.g. in [9, Theorem 2.5.14]. In the present paper, we extend the proof to the whole Euclidean plane.

In the next section, we introduce notation and write down equations of motion with boundary conditions. Afterwards we consider infinite, open, and closed strings in subsequent sections, respectively. Finally, we analyze the Riemannian two-dimensional metric.

All functions are supposed to be sufficiently smooth by default.

\section{The bosonic string}

Consider two manifolds: a plane $\mathbb{R}^{2}$ with arbitrary global coordinates $x=\left(x^{\alpha}\right):=\left(x^{0}, x^{1}\right):=(\tau, \sigma), \alpha=0,1$, and $D$-dimensional Minkowskian space $\mathbb{R}^{1, D-1}$ with Cartesian 
coordinates $X=\left(X^{\mathrm{A}}\right), \mathrm{A}=0,1, \ldots, D-1, D \geq 2$, and the Lorentz metric $\eta_{\mathrm{AB}}:=\operatorname{diag}(+-\cdots-)$. Let there be a smooth embedding

$$
X: \quad \mathbb{R}^{2} \supset \overline{\mathbb{U}} \ni \quad(\tau, \sigma) \mapsto\left(X^{\mathrm{A}}(\tau, \sigma)\right) \quad \in \mathbb{R}^{1, D-1},
$$

of some closed subset $\overline{\mathbb{U}}$ of a plane. We assume that $\mathbb{U}$ is connected and simply connected open subset in $\mathbb{R}^{2}$. The embedding defines the string worldsheet $\mathbb{M}:=X(\overline{\mathbb{U}})$.

Embedding (1) defines symmetric quadratic form with components

$h_{\alpha \beta}:=\partial_{\alpha} X^{\mathrm{A}} \partial_{\beta} X^{\mathrm{B}} \eta_{\mathrm{AB}}=\partial_{\alpha} X^{\mathrm{A}} \partial_{\beta} X_{\mathrm{A}}$.

In general, this form may be negative definite, degenerate, or indefinite. We assume that the embedding is such that

$$
\begin{aligned}
& \left(\partial_{0} X\right)^{2}:=\dot{X}^{2}:=\dot{X}^{\mathrm{A}} \dot{X}^{\mathrm{B}} \eta_{\mathrm{AB}}>0, \\
& \left(\partial_{1} X\right)^{2}:=X^{\prime 2}:=X^{\prime \mathrm{A}} X^{\prime \mathrm{B}} \eta_{\mathrm{AB}}<0,
\end{aligned}
$$

where the dot and prime denote differentiation with respect to $\tau$ and $\sigma$, respectively, on $\mathbb{U}$. The vectors $\dot{X}$ and $X^{\prime}$ are linearly independent on $\mathbb{M}$. Here and in what follows indices A, B, .. are often omitted. So global coordinates $\tau, \sigma$ on $\mathbb{U}$ are timelike and spacelike, respectively. Then the determinant of the induced quadratic form is negative

$h:=\operatorname{det} h_{\alpha \beta}=\dot{X}^{2} X^{\prime 2}-\left(\dot{X}, X^{\prime}\right)^{2}<0$,

where brackets denote the usual scalar product in $\mathbb{R}^{1, D-1}$. Now the embedding (1) defines the Lorentzian metric on the string worldsheet interior $\mathbb{U}$ with signature $(+-)$.

Open string is the embedding (1) of the closed straight strip

$-\infty<\tau<\infty, \quad 0 \leq \sigma \leq \pi$

with properties (3). This strip is vertical if $\tau$ and $\sigma$ coordinate axes are depicted by vertical and horizontal straight lines on a plain $\mathbb{R}^{2}$, respectively.

Closed string is the embedding (1) of the closed straight vertical strip

$$
-\infty<\tau<\infty, \quad-\pi \leq \sigma \leq \pi
$$

with identified boundaries. There are many ways to identify smoothly the boundaries (6). In string theory, we, first, impose the conformal gauge on the metric on the same strip (6) and, second, impose the smooth periodicity conditions

$\left.\partial_{1}^{k} X^{\mathrm{A}}\right|_{\sigma=-\pi}=\left.\partial_{1}^{k} X^{\mathrm{A}}\right|_{\sigma=\pi}, \quad \forall \mathrm{A}, \forall \tau, \quad k=0,1,2, \ldots,(7)$

up to the needed order. It is the prime aim of the present paper to prove that the conformal gauge on the same strips does exist.

Sure, a cylinder is not a simply connected manifold and cannot be covered by a single coordinate chart. The domain
(6) is the fundamental domain for a closed string worldsheet with identified boundaries.

A coordinate system defined on the domains for open (5) and for closed (6) strings we call global coordinate system on the string worldsheets.

If infinite strips in the $\tau, \sigma$ plane have curved boundaries, then all of them are diffeomorphic to strips (5) or (6). Thus we did not loose generality by specifying the coordinate range in the $\tau, \sigma$ plane.

The dynamics of the Nambu-Goto string is governed by the action which is proportional to the string worldsheet area

$S_{\mathrm{NG}}:=-\int_{\overline{\mathbb{U}}} d x \sqrt{|h|}=-\int_{\overline{\mathbb{U}}} d \tau d \sigma \sqrt{\left(\dot{X}, X^{\prime}\right)^{2}-\dot{X}^{2} X^{\prime 2}}$,

where $h:=\operatorname{det} h_{\alpha \beta}$. This action is invariant with respect to arbitrary coordinate changes and global Lorentz transformations. It implies the Euler-Lagrange equations

$$
\begin{aligned}
\frac{1}{\sqrt{|h|} \frac{\delta S_{\mathrm{NG}}}{\delta X_{\mathrm{A}}}} & =\square_{(h)} X^{\mathrm{A}}=h^{\alpha \beta} \nabla_{\alpha} \nabla_{\beta} X^{\mathrm{A}} \\
& =\frac{1}{\sqrt{|h|}} \partial_{\alpha}\left(\sqrt{|h|} h^{\alpha \beta} \partial_{\beta} X^{\mathrm{A}}\right)=0,
\end{aligned}
$$

where the two-dimensional wave operator $\square_{(h)}$ is build by the induced metric $h_{\alpha \beta}$ (2) and $\nabla_{\alpha}$ is the covariant derivative with respective Christoffel's symbols.

We assume that ends of an open string are free, and then the action (8) implies also the boundary conditions

$\left.s^{\beta} \partial_{\beta} X^{\mathrm{A}}\right|_{\sigma=0, \pi}=0$,

where $s^{\alpha}$ are components of the spacelike vector which is perpendicular to the boundaries with respect to the induced metric.

The action (8) does not yield any boundary condition for a closed string. Instead, we have periodicity conditions (7) imposed by hands.

In string theory, the crucial role is played by the possibility to impose global conformal gauge

$h_{\alpha \beta}=\mathrm{e}^{2 \phi} \eta_{\alpha \beta}, \quad \eta_{\alpha \beta}:=\operatorname{diag}(+-)$,

where $\phi(x)$ is some sufficiently smooth function, on the whole string worldsheet. The aim of the present paper is to prove that this conformal gauge can be imposed on the same strips (5) and (6) both for open and closed strings with the same straight boundaries.

\section{The idea of the proof}

The idea of the proof is the following. We construct two orthogonal vector fields: the timelike $t=t^{\alpha} \partial_{\alpha}$ and spacelike $s=s^{\alpha} \partial_{\alpha}$ vector fields such that the following conditions hold on the whole string worldsheet $\mathbb{U} \hookrightarrow \mathbb{R}^{1, D-1}$ : 


$$
(t, s)=0, \quad t^{2}+s^{2}=0, \quad t^{2}>0, \quad \forall x \in \mathbb{U},
$$

where the scalar product is defined by the induced metric $h_{\alpha \beta}$ (2):

$$
(t, s):=t^{\alpha} s^{\beta} h_{\alpha \beta}, \quad t^{2}:=(t, t), \quad s^{2}:=(s, s) .
$$

Then we find conditions for commutativity of these vector fields: $[t, s]=0$. The next step is to find two families of integral curves $x^{\alpha}(\tilde{\tau}, \tilde{\sigma})$ which are defined by the system of differential equations

$\frac{\partial x^{\alpha}}{\partial \tilde{\tau}}=t^{\alpha}, \quad \frac{\partial x^{\alpha}}{\partial \tilde{\sigma}}=s^{\alpha}$,

where $\tilde{\tau}$ and $\tilde{\sigma}$ are parameters along integral curves of vector fields $t$ and $s$, respectively. The integrability conditions for this system are fulfilled on the whole $\mathbb{U}$ :

$$
\begin{aligned}
\frac{\partial^{2} x^{\alpha}}{\partial \tilde{\tau} \partial \tilde{\sigma}}-\frac{\partial^{2} x^{\alpha}}{\partial \tilde{\sigma} \partial \tilde{\tau}} & =\frac{\partial s^{\alpha}}{\partial \tilde{\tau}}-\frac{\partial t^{\alpha}}{\partial \tilde{\sigma}}=t^{\beta} \partial_{\beta} s^{\alpha}-s^{\beta} \partial_{\beta} t^{\alpha} \\
& =[t, s]^{\alpha}=0
\end{aligned}
$$

due to commutativity of vector fields. Consequently, there is a nondegenerate coordinate transformation $(\tau, \sigma) \mapsto(\tilde{\tau}, \tilde{\sigma})$ on the whole worldsheet $\mathbb{U}$.

In the new coordinate system, the induced metric $\tilde{h}_{\alpha \beta}$ is conformally flat due to the properties of the vector fields (12):

$$
\begin{aligned}
& \tilde{h}_{00}=h_{\alpha \beta} \frac{\partial x^{\alpha}}{\partial \tilde{\tau}} \frac{\partial x^{\beta}}{\partial \tilde{\tau}}=t^{2}, \\
& \tilde{h}_{01}=h_{\alpha \beta} \frac{\partial x^{\alpha}}{\partial \tilde{\tau}} \frac{\partial x^{\beta}}{\partial \tilde{\sigma}}=(t, s)=0, \\
& \tilde{h}_{11}=h_{\alpha \beta} \frac{\partial x^{\alpha}}{\partial \tilde{\sigma}} \frac{\partial x^{\beta}}{\partial \tilde{\sigma}}=s^{2}=-t^{2} .
\end{aligned}
$$

The final step is the analysis of domains of the definition of parameters $\tilde{\tau}$ and $\tilde{\sigma}$ of integral curves (13) which are new coordinates.

The last two conditions (12) imply the inequality $s^{2}<0$, i.e. the vector field $s$ is necessarily spacelike.

Here and in what follows, $\mathbb{U}$ denotes either the whole Euclidean plane (infinite string) or an open set (strip) on the plane $(\tau, \sigma) \in \mathbb{U} \subset \mathbb{R}^{2}$ (open or closed string), where the induced metric is nondegenerate. The boundaries $\partial \mathbb{U}$ of open string, on which the metric is degenerate, are considered separately.

\section{Infinite string}

Let us start the detailed analysis. First, we consider the embedding (1) where $\mathbb{U}=\mathbb{R}^{2}$, i.e. the embedding of the whole plane (infinite string). Arbitrary timelike and spacelike tangent vectors $T$ and $S$ to the string worldsheet in the embedding space $\mathbb{R}^{1, D-1}$ can be decomposed on tangent vectors $\dot{X}$ and $X^{\prime}$ :

$$
\begin{aligned}
T & =A\left(\cosh \varphi \dot{X}+\sinh \varphi X^{\prime}\right), \\
S & =B\left(\sinh \psi \dot{X}+\cosh \psi X^{\prime}\right),
\end{aligned}
$$

where $A(x) \neq 0, B(x) \neq 0$ and $\varphi(x), \psi(x) \in \mathbb{R}$ are some functions. Suppose that vectors $T$ and $S$ for $\varphi=\psi=0$ are directed in the same way as vectors $\dot{X}$ and $X^{\prime}$, respectively. Then $A>0$ and $B>0$.

Lemma 4.1 Vector fields $T$ and $S$ on $\mathbb{U}$ satisfy equalities

$(T, S)=0, \quad T^{2}+S^{2}=0$,

if and only if vector field $S$ is given by Eq. (15) with arbitrary functions $B>0$ and $\psi \in \mathbb{R}$, and the second vector field has the form

$$
\begin{aligned}
T= & -\frac{B}{\sqrt{|h|}}\left[\cosh \psi X^{\prime 2}+\sinh \psi\left(\dot{X}, X^{\prime}\right)\right] \dot{X} \\
& +\frac{B}{\sqrt{|h|}}\left[\sinh \psi \dot{X}^{2}+\cosh \psi\left(\dot{X}, X^{\prime}\right)\right] X^{\prime} .
\end{aligned}
$$

Proof Substitution of Eq. (15) into the orthogonality condition (16) yields equation

$\begin{aligned} \frac{(T, S)}{\cosh \varphi \cosh \psi}= & \tanh \psi \dot{X}^{2}+(1+\tanh \varphi \tanh \psi)\left(\dot{X}, X^{\prime}\right) \\ & +\tanh \varphi X^{\prime 2}=0\end{aligned}$

which imply

$\tanh \varphi=-\frac{\tanh \psi \dot{X}^{2}+\left(\dot{X}, X^{\prime}\right)}{X^{\prime 2}+\tanh \psi\left(\dot{X}, X^{\prime}\right)}$.

Then vector field $T$ can be written in the form

$$
\begin{aligned}
T= & -\tilde{A}\left[X^{\prime 2}+\tanh \psi\left(\dot{X}, X^{\prime}\right)\right] \dot{X} \\
& +\tilde{A}\left[\tanh \psi \dot{X}^{2}+\left(\dot{X}, X^{\prime}\right)\right] X^{\prime},
\end{aligned}
$$

where

$A:=\tilde{A} \sqrt{\left[\tanh \psi \dot{X}^{2}+\left(\dot{X}, X^{\prime}\right)\right]^{2}+\left[X^{\prime 2}+\tanh \psi\left(\dot{X}, X^{\prime}\right)\right]^{2}}$.

Now algebraic equation $T^{2}+S^{2}=0$ has the unique solution $\tilde{A}=\frac{B}{\sqrt{|h|}} \cosh \psi$.

As a result, we obtain solution (17) for arbitrary $S$.

The similar statement can be formulated considering the vector field $T$ as independent variable. To this end we have to solve Eq. (18) with respect to $\psi$ and afterwards find $S$.

Vector fields $T$ and $S$ are defined on the whole string worldsheet and lie in the tangent space to the Minkowskian space $\mathbb{T}\left(\mathbb{R}^{1, D-1}\right)$. The differential map of the embedding $\mathbb{U} \hookrightarrow \mathbb{R}^{1, D-1}$ acts on vectors as follows 


$$
\begin{aligned}
\mathbb{T}(\mathbb{U}) \ni \quad t & =t^{\alpha} \partial_{\alpha}, s=s^{\alpha} \partial_{\alpha} \mapsto \\
T & =t^{\alpha} \partial_{\alpha} X^{\mathrm{A}} \partial_{\mathrm{A}}, S=s^{\alpha} \partial_{\alpha} X^{\mathrm{A}} \partial_{\mathrm{A}} \in \mathbb{T}\left(\mathbb{R}^{1, D-1}\right),
\end{aligned}
$$

where $t$ and $s$ are vector fields on $\mathbb{U}$. Note the relations

$T^{2}=t^{2} \quad S^{2}=s^{2}, \quad(T, S)=(t, s)$,

which follow from the definition of the induced metric. Comparing the above formulae with Eqs. (15) and (17) allows us to define vector fields on $\mathbb{U}$ :

$$
\begin{aligned}
t= & -\frac{B}{\sqrt{|h|}}\left[\cosh \psi X^{\prime 2}+\sinh \psi\left(\dot{X}, X^{\prime}\right)\right] \partial_{0} \\
& +\frac{B}{\sqrt{|h|}}\left[\sinh \psi \dot{X}^{2}+\cosh \psi\left(\dot{X}, X^{\prime}\right)\right] \partial_{1} . \\
s= & B \sinh \psi \partial_{0}+B \cosh \psi \partial_{1} .
\end{aligned}
$$

These vectors can be easily rewritten in the form

$t=\varepsilon^{\alpha \beta} s_{\beta} \partial_{\alpha}, \quad s=s^{\alpha} \partial_{\alpha}$,

where $\varepsilon^{\alpha \beta}$ is the totally antisymmetric second rank tensor, $\varepsilon^{01}=-1 / \sqrt{|h|}$, and components $s^{\alpha}$ are arbitrary. It immediately implies equalities (12). There is a one-to-one correspondence between vector components (21)

$t^{\alpha}=\varepsilon^{\alpha \beta} s_{\beta} \quad \Leftrightarrow \quad s_{\alpha}=\varepsilon_{\alpha \beta} t^{\beta}$.

That is we can take either $t^{\alpha}$ or $s^{\alpha}$ as independent variables. Now we have to find the condition of their commutativity.

Lemma 4.2 Vector fields $t$ and $s$ on $\mathbb{U}$ related by equalities (22) commute if and only if

$t_{\alpha}=\frac{\partial_{\alpha} \chi}{\partial \chi^{2}}, \quad \partial \chi^{2}:=h^{\alpha \beta} \partial_{\alpha} \chi \partial_{\beta} \chi>0$,

where $\chi$ is a nontrivial solution of the wave equation

$\square_{(h)} \chi:=h^{\alpha \beta} \nabla_{\alpha} \nabla_{\beta} \chi=0$,

satisfying $\partial \chi^{2}>0$.

For any nontrivial solution of Eq. (24) satisfying the condition $\partial \chi^{2}>0$, vector fields (22) and (23) commute.

Note that for different nontrivial solutions of the wave equation (24) the pairs of vector fields $t, s$ differ in general.

Proof The equality $[t, s]=0$ together with conditions (22) is equivalent to the system of equations

$s_{\alpha} \nabla_{\beta} s^{\beta}-\frac{1}{2} \nabla_{\alpha} s^{2}-s^{\beta} \nabla_{\beta} s_{\alpha}=0$.

This equation is equivalent to the same equation for $t$ :

$t_{\alpha} \nabla_{\beta} t^{\beta}-\frac{1}{2} \nabla_{\alpha} t^{2}-t^{\beta} \nabla_{\beta} t_{\alpha}=0$.

Contraction of Eq. (25) with $s^{\alpha}$ yields

$\nabla_{\alpha}\left(\frac{s^{\alpha}}{s^{2}}\right)=0 \quad \Leftrightarrow \quad \varepsilon^{\alpha \beta} \nabla_{\alpha}\left(\frac{t_{\beta}}{t^{2}}\right)=0$.
Since Christoffel's symbols are symmetric, the covariant derivatives in the last equation can be replaced by the partial ones. Then, due to the Poincaré lemma, there exists such function $\chi$ that

$\frac{t_{\alpha}}{t^{2}}=\partial_{\alpha} \chi \quad \Rightarrow \quad t^{2}=\frac{1}{\partial \chi^{2}}$

on arbitrary connected and simply connected domain $\mathbb{U}$, in particular, on the whole plane $\mathbb{U}=\mathbb{R}^{2}$.

We require $\partial \chi^{2}>0$ because vector field $t$ on $\mathbb{U}$ must be timelike, $t^{2}>0$. Consequently, representation (23) is valid for components $t_{\alpha}$. Substitution of equality (23) into Eq. (26) yields

$\frac{\partial_{\alpha} \chi \square_{(h)} \chi}{\partial \chi^{2}}=0$

Since $\left(\partial_{\alpha} \chi\right) \neq 0$ and $\partial \chi^{2}>0$, we obtain Eq. (24) for the unknown function $\chi$.

It is clear that for any nontrivial solution of the wave equation (24) the vector fields $t$ and $s$ exist, commute, and have properties (12).

Thus commuting vector fields $t$ and $s$ with properties (12) have generally the following form

$t=\frac{h^{\alpha \beta} \partial_{\beta} \chi}{\partial \chi^{2}} \partial_{\alpha}, \quad s=\frac{\varepsilon^{\alpha \beta} \partial_{\beta} \chi}{\partial \chi^{2}} \partial_{\alpha}$,

where $\chi$ is an arbitrary solution of the wave equation (24) such that $\partial \chi^{2}>0$. That is we have described the total arbitrariness existing in vector fields if Eq. (24) has many solutions.

Comment We did not use in Lemma 4.2 the fact that vector fields $t$ and $s$ were obtained by the embedding $U \hookrightarrow \mathbb{R}^{1, D-1}$. It is sufficient to consider two vector fields related by Eq. (21). The properties (12) are easily verified without embedding. $\square$

Suppose that the determinant of the induced metric $h_{\alpha \beta}$ is nonzero on the whole plane $(\tau, \sigma) \in \mathbb{R}^{2}$ and separated from 0 and $\pm \infty$ at infinity:

$0<\epsilon \leq \lim _{\tau^{2}+\sigma^{2} \rightarrow \infty}\left|\operatorname{det} h_{\alpha \beta}\right| \leq M<\infty$,

where $\epsilon$ and $M$ are some constants. It is well known that the Cauchy problem for the hyperbolic equation (24) has unique solution $\chi$ on the whole plain, if the Cauchy data are given on a spacelike curve, say, $\tau=0$ (see, e.g. [7], book IV, ch. I). It is easily verified that there exist such Cauchy data that the inequality $\partial \chi^{2}>0$ holds everywhere. This implies that nontrivial solution of the wave equation (24) exists on the whole plane $\mathbb{R}^{2}$. There are many such solutions, and they are parameterized by the Cauchy data.

Thus the vector fields $s$ and $t$ are given on the whole plane $\mathbb{R}^{2}$. The inequality (30) implies that component $t^{0}$ is separated from zero and bounded on the plane including infinity. 
Thus Eq. (13) imply

$$
\frac{\partial \tau}{\partial \tilde{\tau}}=t^{0} \quad \Rightarrow \quad \tilde{\tau} \sim \int^{\infty} \frac{d \tau}{t^{0}} .
$$

Example 1 Let the induced metric be conformally flat:

$h_{\alpha \beta} d x^{\alpha} d x^{\beta}=\mathrm{e}^{2 \phi}\left(d \tau^{2}-d \sigma^{2}\right)=\mathrm{e}^{2 \phi} d \xi d \eta, \quad \phi=\phi(x)$,

The last integral is divergent and thus the coordinate $\tilde{\tau}$ runs over the whole real line $\mathbb{R}$. Similar statement is valid for the space coordinate $\tilde{\sigma}$. Consequently, new coordinates cover the whole plane $(\tilde{\tau}, \tilde{\sigma}) \in \mathbb{R}^{2}$.

Thus we proved

Theorem 4.1 Let an arbitrary metric $h_{\alpha \beta}$ of Lorentzian signature be given on the whole plane $\mathbb{R}^{2}$. Let it be nondegenerate at infinity (30). Then there exists a surjective diffeomorphism on the plane

$\mathbb{R}^{2} \ni \quad\left(x^{\alpha}\right) \mapsto\left(\tilde{x}^{\alpha}(x)\right) \in \mathbb{R}^{2}$

such that metric $h_{\alpha \beta}$ in new coordinate system has conformally flat form

$\tilde{h}_{\alpha \beta}:=h_{\gamma \delta} \frac{\partial x^{\gamma}}{\partial \tilde{x}^{\alpha}} \frac{\partial x^{\delta}}{\partial \tilde{x}^{\beta}}=\mathrm{e}^{2 \phi} \eta_{\alpha \beta}$

where $\phi(\tilde{x})$ is some function on $\mathbb{R}^{2}$ separated from $\pm \infty$ at infinity $\tilde{\tau}^{2}+\tilde{\sigma}^{2} \rightarrow \infty$.

In contrast to the local theorem (see, e.g., $[4,5])$ stating the existence of the conformal gauge only in some neighborhood of every point, the above theorem is global in a sense that it provides the existing of the conformal gauge for a Lorentzian metric given on the whole plane $x \in \mathbb{R}^{2}$.

If metric $h_{\alpha \beta}$ and scalar field $\chi$ are given, then the diffeomorphism (31) is defined uniquely up to shifts of coordinates $\tilde{\tau}$ and $\tilde{\sigma}$ (constants of integration of Eq. (13)).

\section{Corollary Let}

$\widetilde{\mathbb{U}}_{0}:=\left\{(\tilde{\tau}, \tilde{\sigma}) \in \mathbb{R}^{2}: \quad \tilde{\sigma} \in\left[\tilde{\sigma}_{1}, \tilde{\sigma}_{2}\right], \tilde{\tau} \in \mathbb{R}\right\}$

be closed vertical strip with straight boundaries on the plane of new coordinates $\tilde{\tau}, \tilde{\sigma}$ and assumptions of Theorem 4.1 hold. Then there exists diffeomorphism (31) of a closed domain $(\tau, \sigma) \in \overline{\mathbb{U}} \subset \mathbb{R}^{2}$ bounded by integral curves $x\left(\tilde{\tau}, \tilde{\sigma}_{1,2}\right)$ :

$\frac{\partial x\left(\tilde{\tau}, \tilde{\sigma}_{1,2}\right)}{\partial \tilde{\tau}}=t_{1,2}$,

where $t_{1,2}$ are inverse images of vector fields $\partial / \partial \tilde{\tau}$ on the boundaries of $\widetilde{\mathbb{U}}_{0}$.

Proof Internal and boundary points are mapped under diffeomorphism into internal and boundary points, respectively.

To clarify the arbitrariness in coordinates $\tilde{\tau}, \tilde{\sigma}$ defined by the function $\chi$ we consider where light cone coordinates $\xi:=\tau+\sigma, \eta:=\tau-\sigma$ are introduced. Then the wave equation (24) is reduced to the flat d'Alembert equation

$\square_{(h)} \chi=\left(\partial_{0}^{2}-\partial_{1}^{2}\right) \chi=0$.

Its general solution is given by two arbitrary sufficiently smooth functions

$\chi=F(\xi)+G(\eta)$.

We choose only the functions which satisfy inequality

$\partial \chi^{2}=4 \mathrm{e}^{-2 \phi} F^{\prime} G^{\prime}>0 \quad \Rightarrow \quad F^{\prime} G^{\prime}>0$,

where prime denotes differentiation by the corresponding argument. Then the metric takes the form

$\tilde{h}_{\alpha \beta} d \tilde{x}^{\alpha} d \tilde{x}^{\beta}=\frac{\mathrm{e}^{2 \phi}}{4 F^{\prime} G^{\prime}} d \tilde{\xi} d \tilde{\eta}$

in new coordinates $\tilde{\xi}:=\tilde{\tau}+\tilde{\sigma}, \tilde{\eta}:=\tilde{\tau}-\tilde{\sigma}$. This metric corresponds to the conformal transformation

$\tilde{\xi}:=2 F(\xi), \quad \tilde{\eta}:=2 G(\eta)$

Thus the arbitrariness in definition of the vector fields described in Lemma 4.2 corresponds to conformal transformations on the string worldsheet.

Let us find the image of vector fields $t$ and $s$ (29) under the coordinate transformation $x^{\alpha} \mapsto \tilde{x}^{\alpha}(x)$ where $\left(\tilde{x}^{\alpha}\right)=$ $(\tilde{\tau}, \tilde{\sigma})$. The definition of new coordinates (13) implies the expression for the inverse Jacobi matrix

$J^{-1}{ }_{\alpha}^{\beta}=\frac{\partial x^{\beta}}{\partial \tilde{x}^{\alpha}}=\left(\begin{array}{ll}t^{0} & t^{1} \\ s^{0} & s^{1}\end{array}\right)$.

This expression yields the Jacobian of the coordinate transformation

$J:=\operatorname{det} J_{\alpha}{ }^{\beta}=\left(t^{0} s^{1}-t^{1} s^{0}\right)^{-1}=\sqrt{|h|} \partial \chi^{2}$,

where representation (29) is used, and the Jacobi matrix

$J_{\alpha}{ }^{\beta}:=\frac{\partial \tilde{x}^{\beta}}{\partial x^{\alpha}}=\sqrt{|h|} \partial \chi^{2}\left(\begin{array}{cc}s^{1} & -t^{1} \\ -s^{0} & t^{0}\end{array}\right)$.

Vector fields are transformed under the differential of the map:

$t^{\alpha} \partial_{\alpha} \mapsto \tilde{t}^{\alpha} \tilde{\partial}_{\alpha}:=t^{\beta} \partial_{\beta} \tilde{x}^{\alpha} \tilde{\partial}_{\alpha}=\partial_{\tilde{\tau}}$,

$s^{\alpha} \partial_{\alpha} \mapsto \tilde{s}^{\alpha} \tilde{\partial}_{\alpha}:=s^{\beta} \partial_{\beta} \tilde{x}^{\alpha} \tilde{\partial}_{\alpha}=\partial_{\tilde{\sigma}}$.

It means that integral curves of the vector fields $t$ and $s$ are perpendicular straight lines on the plane $(\tilde{\tau}, \tilde{\sigma}) \in \mathbb{R}^{2}$. 
In the target Minkowskian space $\mathbb{R}^{1, D-1}$ the vector fields $t$ and $s$ have the form

$$
\begin{gathered}
T=\frac{h^{\alpha \beta} \partial_{\beta} \chi}{\partial \chi^{2}} \partial_{\alpha} X=\frac{h^{0 \beta} \partial_{\beta} \chi}{\partial \chi^{2}} \dot{X}+\frac{h^{1 \beta} \partial_{\beta} \chi}{\partial \chi^{2}} X^{\prime}, \\
S=\frac{\varepsilon^{\alpha \beta} \partial_{\beta} \chi}{\partial \chi^{2}} \partial_{\alpha} X=\frac{\varepsilon^{0 \beta} \partial_{\beta} \chi}{\partial \chi^{2}} \dot{X}+\frac{\varepsilon^{1 \beta} \partial_{\beta} \chi}{\partial \chi^{2}} X^{\prime} .
\end{gathered}
$$

It was already mentioned, that the wave equation (24) has nontrivial solutions $\chi, \partial \chi^{2} \neq 0$, on the whole plane $(\tau, \sigma) \in \mathbb{R}^{2}$. The wave equation has the same form in new coordinates $\tilde{\tau}, \tilde{\sigma}$ as on the Minkowskian plane. Therefore a general solution of the wave equation (24) is

$$
\begin{gathered}
\chi=F(\tilde{\xi})+G(\tilde{\eta})=F(\tilde{\tau}+\tilde{\sigma})+G(\tilde{\tau}-\tilde{\sigma}), \\
(\tilde{\xi}, \tilde{\eta}) \in \mathbb{R}^{2},
\end{gathered}
$$

where $F$ and $G$ are two arbitrary sufficiently smooth functions of one argument. It implies equalities:

$$
\begin{aligned}
& \partial_{0} \chi:=\frac{\partial \chi}{\partial \tau}=\sqrt{|h|} \partial \chi^{2}\left[\left(s^{1}-t^{1}\right) F^{\prime}+\left(s^{1}+t^{1}\right) G^{\prime}\right], \\
& \partial_{1} \chi:=\frac{\partial \chi}{\partial \sigma}=\sqrt{|h|} \partial \chi^{2}\left[\left(t^{0}-s^{0}\right) F^{\prime}+\left(t^{0}+s^{0}\right) G^{\prime}\right],
\end{aligned}
$$

were Jacobi's matrices (36) are used. Then the components of vectors $\hat{t}$ and $\hat{s}$ corresponding to solution (38) are

$$
\begin{aligned}
\hat{t}^{0}= & -\frac{1}{\sqrt{|h|}}\left[X^{\prime 2}\left(s^{1}-t^{1}\right) F^{\prime}+X^{\prime 2}\left(s^{1}+t^{1}\right) G^{\prime}\right. \\
& \left.+\left(\dot{X}, X^{\prime}\right)\left(s^{0}-t^{0}\right) F^{\prime}+\left(\dot{X}, X^{\prime}\right)\left(s^{0}+t^{0}\right) G^{\prime}\right], \\
\hat{t}^{1}= & \frac{1}{\sqrt{|h|}}\left[\left(\dot{X}, X^{\prime}\right)\left(s^{1}-t^{1}\right) F^{\prime}+\left(\dot{X}, X^{\prime}\right)\left(s^{1}+t^{1}\right) \underset{(39)}{G^{\prime}}\right. \\
& \left.+\dot{X}^{2}\left(s^{0}-t^{0}\right) F^{\prime}+\dot{X}^{2}\left(s^{0}+t^{0}\right) G^{\prime}\right], \\
\hat{s}^{0}= & \left(s^{0}-t^{0}\right) F^{\prime}+\left(s^{0}+t^{0}\right) G^{\prime}, \\
\hat{s}^{1}= & \left(s^{1}-t^{1}\right) F^{\prime}+\left(s^{1}+t^{1}\right) G^{\prime} .
\end{aligned}
$$

The components on the left hand side are marked by the hat because vectors $\hat{t}$ and $\hat{s}$ differ in general from the ones which were used for the transformation of the wave equation to the flat form. However for some functions $F$ and $G$ we must get identities.

\section{Proposition 4.1 Equalities}

$\hat{t}=t, \quad \hat{s}=s$

become identities if and only if arbitrary functions in solution (38) are linear: $F^{\prime}=G^{\prime}=1 / 2$.

Proof Straightforward calculations.

Thus for

$\chi=\frac{1}{2} \tilde{\xi}+\frac{1}{2} \tilde{\eta}=\tilde{\tau}$, equalities (29) become identities. The vector fields $\partial_{\tilde{\tau}}$ and $\partial_{\tilde{\sigma}}$ are obtained from the fields $t$ and $s$ by the differential of the map $(\tau, \sigma) \mapsto(\tilde{\tau}, \tilde{\sigma})$ only for this solution of the wave equation.

Thus to find the diffeomorphism (31) in explicit form for a given metric $h_{\alpha \beta}$, we have to (i) find a nontrivial solution of the wave equation (24), (ii) construct the vector fields $t$ and $s$ using Eqs. (22), (23), and (iii) find a general solution of the system of equations (13). We have proved that this problem does have many solutions (the whole arbitrariness is contained in the choice of nontrivial solution of the wave equation).

\section{Open string}

Now we consider an open string whose worldsheet $\overline{\mathbb{U}}$ is an infinite strip on the plane $(\tau, \sigma) \in \mathbb{R}^{2}$ with two, probably, curved boundaries: the left $\gamma_{L}$ and right $\gamma_{R}$ boundaries. The induced metric on the boundaries is degenerate, and results of the previous section must be revised. First, we assume that metric is not degenerate and return to this problem later.

If the metric is nondegenerate on $\overline{\mathbb{U}}$ including the boundaries, then we continue it on the whole plain in some sufficiently smooth manner. As the consequence of theorem 4.1 there is a diffeomorphism (31) after which the metric becomes conformally flat. The problem is that the boundaries $\gamma_{\mathrm{L}, \mathrm{R}}$ on the plain $\tilde{\tau}, \tilde{\sigma}$ may be not straight vertical lines. However there are residual diffeomorphisms in the form of conformal maps of $\tilde{\tau}, \tilde{\sigma}$ coordinates. We now show that it is enough to straighten the strip.

Remember that we do not consider shifts of the plain $\tilde{\xi}, \tilde{\eta} \in \mathbb{R}^{2}$ as a whole which preserve the conformal form of the metric but is not a conformal map.

Let boundary equations after diffeomorphism $(\tau, \sigma) \mapsto$ $(\tilde{\tau}, \tilde{\sigma})$ be (see Fig. 1)

$\gamma_{\mathrm{L}}: \quad \tilde{\eta}=\tilde{\eta}_{\mathrm{L}}(\tilde{\xi}), \quad \gamma_{\mathrm{R}}: \quad \tilde{\eta}=\tilde{\eta}_{\mathrm{R}}(\tilde{\xi}), \quad \tilde{\xi} \in \mathbb{R}$,

where functions $\tilde{\eta}_{\mathrm{L}, \mathrm{R}} \in \mathcal{C}^{1}(\mathbb{R})$ have properties:

$\tilde{\eta}_{\mathrm{L}}>\tilde{\eta}_{\mathrm{R}}, \quad 0<\epsilon \leq \frac{d \tilde{\eta}_{\mathrm{L}, \mathrm{R}}}{d \tilde{\xi}} \leq M<\infty, \quad \epsilon, M \in \mathbb{R}$

for all $\tilde{\xi} \in \mathbb{R}$ including infinite points.

\section{Theorem 5.1 The conformal transformation}

$\hat{\xi}=F(\tilde{\xi}), \quad \hat{\eta}=G(\tilde{\eta}), \quad F, G \in \mathcal{C}^{1}(\mathbb{R})$,

such that the boundaries (41) of an open string worldsheet become straight vertical lines

$\gamma_{\mathrm{L}}: \hat{\eta}=\hat{\xi}, \quad \gamma_{\mathrm{R}}: \hat{\eta}=\hat{\xi}-2 \pi, \quad \hat{\xi} \in \mathbb{R}$

on the plain $\hat{\xi}, \hat{\eta} \in \mathbb{R}^{2}$ exists. 
Fig. 1 Open string worldsheet in different coordinates $(\tau, \sigma)$, $(\tilde{\tau}, \tilde{\sigma})$, and $(\hat{\tau}, \hat{\sigma})$. Three integral curves are shown both for timelike $t$ and spacelike $s$ vector fields

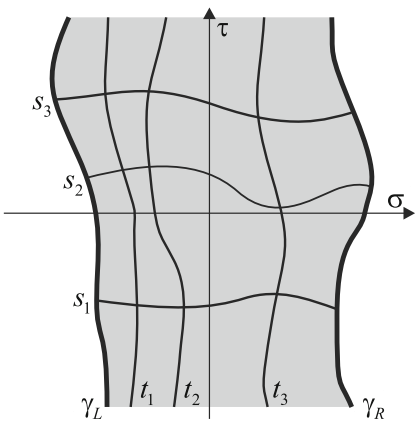

Proof Let us straighten first the left boundary by conformal transformation

$\check{\xi}=F(\tilde{\xi}), \quad \check{\eta}=G(\tilde{\eta})$.

It is necessary and sufficient to fulfill the condition

$F(\tilde{\xi})=G\left(\tilde{\eta}_{\mathrm{L}}(\tilde{\xi})\right), \quad \forall \tilde{\xi} \in \mathbb{R}$

in order that the left boundary to be vertical straight line going through the origin. This equation uniquely defines the function $G$ for a given $F$ because the function $\tilde{\eta}_{\mathrm{L}}$ is strictly monotonic, the function $F$ being arbitrary.

Now we consider the right boundary. After straightening the left boundary, we are left with the coordinate transformation

$\hat{\xi}=F(\check{\xi}), \quad \hat{\eta}=F(\check{\eta})$,

which does not change the left boundary and is described by one arbitrary function $F$. It is necessary and sufficient to satisfy the functional equation

$F\left(\check{\eta}_{\mathrm{R}}(\check{\xi})\right)=F(\check{\xi})-2 \pi, \quad \forall \check{\xi} \in \mathbb{R}$,

after which the right boundary becomes the vertical straight line going through the point $(\hat{\tau}, \hat{\sigma})=(0, \pi)$. This functional equation for $F$ has many solutions. Indeed, the map

$f: \quad \mathbb{R} \ni \quad \check{\xi} \mapsto \check{\eta}_{\mathrm{R}}(\check{\xi}) \quad \in \mathbb{R}$

is a bijective map of real lines, and $\check{\eta}_{\mathrm{R}}(\check{\xi})<\check{\xi}$ for all $\check{\xi} \in \mathbb{R}$. Therefore the sufficiently smooth cyclic group $\left\{f^{k}, k \in \mathbb{Z}\right\}$ is defined. Consequently, the function $F$ with property $F^{\prime}>0$ can be arbitrary defined on the fundamental domain, say, $\left[\check{\eta}_{\mathrm{R}}(0), 0\right]$ and then extended on the whole real line using Eq. (43). If the function satisfies Eq. (43) at the ends of the fundamental domain then we obtain continuous function on $\mathbb{R}$ but its derivative may be discontinuous. The function $F$ must be $\mathcal{C}^{1}$ in order to define the conformal transformation. To avoid possible discontinuities in derivatives, we differentiate Eq. (43):

$F^{\prime}\left(\check{\eta}_{\mathrm{R}}(\check{\xi})\right) \frac{d \check{\eta}_{\mathrm{R}}}{d \check{\xi}}=F^{\prime}(\check{\xi})$.

Since $d \check{\eta} / d \check{\xi} \geq \epsilon>0$, then we define arbitrary the derivative $F^{\prime}>0$ on the fundamental domain $\left[\check{\eta}_{\mathrm{R}}(0), 0\right]$ such that

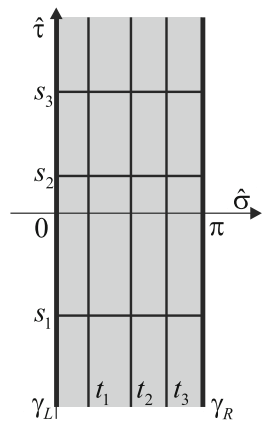

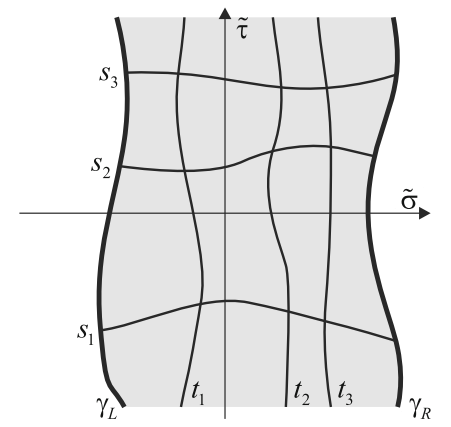

equation

$\left.F^{\prime}\left(\check{\eta}_{\mathrm{R}}(0)\right) \frac{d \check{\eta}_{\mathrm{R}}}{d \check{\xi}}\right|_{\check{\eta}_{\mathrm{R}}(0)}=F^{\prime}(0)$

holds at the ends, and continue it on the real line. Then $F$ is the primitive of $F^{\prime}$ with the constant of integration defined by Eq. (43).

So, if the metric is not degenerate on the boundaries of an open string worldsheet, then there exists such global $\mathcal{C}^{1}$ coordinate transformation that the transformed metric is conformally flat (32) on the whole vertical strip with straight boundaries $\tilde{\sigma}=0$ and $\tilde{\sigma}=\pi$. This statement follows from Theorems 4.1 and 5.1 because the conformal transformation is a diffeomorphism, and diffeomorphisms form a group.

Now we discuss an open Nambu-Goto string for which the induced metric on the boundaries is degenerate due to boundary conditions. Let us parameterize metric $h_{\alpha \beta}$ by its determinant $-\varrho 4$ and "unimodular metric" $k_{\alpha \beta}$ :

$h_{\alpha \beta}:=\varrho^{2} k_{\alpha \beta}, \quad \operatorname{det} k_{\alpha \beta}:=-1, \quad \varrho \geq 0$,

separating its determinant explicitly. For nondegenerate metric $h_{\alpha \beta}$, the inverse transformation is

$\varrho=\left|\operatorname{det} h_{\alpha \beta}\right|^{1 / 4}, \quad k_{\alpha \beta}=\varrho^{-2} h_{\alpha \beta}, \quad \varrho>0$.

Definition (44) implies that the variable $\varrho$ is the scalar density of degree $-1 / 2$ and unimodular metric is the second rank tensor density of degree 1 .

Note that the unimodular metric $k_{\alpha \beta}$ is additionally multiplied by the Jacobian under arbitrary transformation of coordinates because it is a tensor density of degree 1 . It means that the induced and unimodular metrics take the conformally flat form simultaneously.

The boundary condition (10) has the form

$n^{0} \dot{X}^{\mathrm{A}}+n^{1} X^{\prime \mathrm{A}}=0 \quad \Rightarrow \quad X^{\prime \mathrm{A}}=-\frac{n^{0}}{n^{1}} \dot{X}^{\mathrm{A}}$,

because the normal vector to the boundaries $\left(n^{\alpha}\right)=\left(n^{0}, n^{1}\right)$ must be spacelike and consequently $n^{1} \neq 0$. As the consequence, the metric degenerates on the boundaries:

$\operatorname{det} h_{\alpha \beta}=-\rho^{4}=\dot{X}^{2} X^{\prime 2}-\left(\dot{X}, X^{\prime}\right)^{2} \rightarrow 0$. 
Therefore vector fields $t$ and $s$ (12) become null

$t^{2}=\rho^{2} k_{\alpha \beta} t^{\alpha} t^{\beta} \rightarrow 0, \quad s^{2}=\rho^{2} k_{\alpha \beta} s^{\alpha} s^{\beta} \rightarrow 0$

at the ends of the string with respect to the induced metric but not with respect to the unimodular metric, as we shall see later.

Now we construct new vector fields for the unimodular metric $k_{\alpha \beta}$ satisfying relations

$(t, s):=k_{\alpha \beta} t^{\alpha} s^{\beta}=0, \quad t^{2}+s^{2}=0$,

where

$t^{2}:=k_{\alpha \beta} t^{\alpha} t^{\beta} \quad s^{2}:=k_{\alpha \beta} s^{\alpha} s^{\beta}$.

Equalities (45) are equivalent to original equations (12) in internal points of $\mathbb{U}$ and extended on boundaries $\partial \mathbb{U}$ by continuity. It is clear that properties (45) do not contradict Eq. (12). Now we prove that new vector fields $t$ and $s$ exist and coincide with the original ones for $h_{\alpha \beta}$.

Formulae (14) for metric components after the coordinate transformation have the same form. In addition,

$\tilde{h}_{00}=-\tilde{h}_{11}=\varrho^{2} k_{\alpha \beta} t^{\alpha} t^{\beta}, \quad \tilde{h}_{01}=0$.

Equation (28) in new variables does not depend on $\rho$ and therefore function $\chi$ must satisfy equation

$\square_{(k)} \chi:=\partial_{\alpha}\left(k^{\alpha \beta} \partial_{\beta} \chi\right)=0$.

This wave equation has many solutions on the whole plain $\mathbb{R}^{2}$ because depends on nondegenerate unimodular metric $k_{\alpha \beta}$. It implies that vector fields $t$ and $s$ exist and do not depend on $\varrho$ :

$t^{\alpha}=\frac{k^{\alpha \beta} \partial_{\beta} \chi}{k^{\gamma \delta} \partial_{\gamma} \chi \partial_{\delta} \chi} \quad s^{\alpha}=\frac{\hat{\varepsilon}^{\alpha \beta} \partial_{\beta} \chi}{k^{\gamma \delta} \partial_{\gamma} \chi \partial_{\delta} \chi}$,

where

$\hat{\varepsilon}^{\alpha \beta}=\rho^{2} \varepsilon^{\alpha \beta}=\left(\begin{array}{cc}0 & -1 \\ 1 & 0\end{array}\right)$

is the totally antisymmetric tensor density of degree -1 .

One can easily verify that in new variables the EulerLagrange equations for bosonic string (9) take the form

$\sqrt{|h|} \square_{(h)} X^{\mathrm{A}}=\partial_{\alpha}\left(\sqrt{|h|} h^{\alpha \beta} \partial_{\beta} X^{\mathrm{A}}\right)=\partial_{\alpha}\left(k^{\alpha \beta} \partial_{\beta} X^{\mathrm{A}}\right)=0$,

i.e. do not depend on $\varrho$. They must be solved with the boundary condition

$\left.n^{\alpha} \partial_{\alpha} X^{\mathrm{A}}\right|_{\gamma_{\mathrm{L}, \mathrm{R}}}=0$,

which does not depend on $\rho$ too.

Consequently, the problem is reduced to solution of Eq. (48) with boundary conditions (49) for an open NambuGoto string. To make the transformation of coordinates $(\tau, \sigma) \mapsto(\tilde{\tau}, \tilde{\sigma})$ we have to find the unimodular metric $k_{\alpha \beta}$ for a given metric $h_{\alpha \beta}$, choose a solution $\chi$ of the wave equation (46) satisfying the condition $k^{\gamma \delta} \partial_{\gamma} \chi \partial_{\delta} \chi>0$, construct the vector fields $t$ and $s$ using formulae (47), and, finally, integrate Eq. (13). Therefore the corollary of Theorem 4.1 is valid also for an open string. If needed, after solution of this problem, one can compute the conformal factor for the induced metric (32) using equation

$\mathrm{e}^{2 \phi}=\varrho^{2} k_{\alpha \beta} t^{\alpha} t^{\beta}$.

Sure, it is zero on the boundaries because $\rho \rightarrow 0$. Thus we proved the existence of the global conformal gauge for an open string.

\section{Closed string}

In the initial coordinates $\tau, \sigma \in \mathbb{R}^{2}$, the fundamental domain of a closed string worldsheet is given by an infinite strip with timelike boundaries which are identified. The identification can be performed in many ways and therefore requires definition. Here we describe the method adopted in string theory.

We showed in the previous section that there is the global diffeomorphism $(\tau, \sigma) \mapsto(\hat{\tau}, \hat{\sigma})$ which maps an arbitrary infinite strip with timelike boundaries on the vertical strip with straight boundaries where metric becomes conformally flat. The same procedure can be performed for the fundamental domain of a closed string. Without loss of generality, we assume that boundaries go through points $\hat{\sigma}= \pm \pi$, as is usually supposed in string theory. Then the boundary identification is written as the periodicity condition for every value of the timelike coordinate $\hat{\tau}$ :

$$
\left.\frac{\partial^{k} X^{\mathrm{A}}}{\partial \hat{\sigma^{k}}}\right|_{\hat{\sigma}=-\pi}=\left.\frac{\partial^{k} X^{\mathrm{A}}}{\partial \hat{\sigma^{k}}}\right|_{\hat{\sigma}=\pi}, \quad \forall \mathrm{A}, \quad \forall \hat{\tau}, \quad k=0,1,2, \ldots
$$

That is we continuously glue the coordinate functions themselves and their derivatives up to the needed order. In the initial coordinate system this condition is written in the covariant form

$\left.\nabla_{s}^{k} X^{\mathrm{A}}\right|_{\hat{\sigma}=-\pi}=\left.\nabla_{s}^{k} X^{\mathrm{A}}\right|_{\hat{\sigma}=\pi}$,

where $\nabla_{s}:=s^{\alpha} \nabla_{\alpha}$ is the covariant derivative for the LeviCivita connection along the vector field $s$ which is the pullback of the vector field $\partial / \partial \hat{\sigma}$ under the diffeomorphism $(\tau, \sigma) \mapsto(\hat{\tau}, \hat{\sigma})$. The properties of vector fields $t, s(12)$ imply that the covariant derivatives are taken along normal vectors to the boundaries. However it is not clear at the beginning for which value of $\tau$ on the left and right the identification takes place, because we have to find the diffeomorphism $(\tau, \sigma) \mapsto(\hat{\tau}, \hat{\sigma})$ explicitly. Simply speaking we firstly trans- 
form the metric to conformally flat form and afterwards perform the natural gluing.

\section{The Euclidean signature}

Many of the preceeding statements do not depend on the signature of the metric, and the results can be generalized for an arbitrary Riemannian positive definite metric. In this section, we suppose that sufficiently smooth two-dimensional Riemannian metric $h_{\alpha \beta}$, det $h_{\alpha \beta}>0$, is given on the whole plane $\mathbb{R}^{2}$. It is not necessarily induced by some embedding.

Let us consider two vector fields $t=t^{\alpha} \partial_{\alpha}$ and $s=s^{\alpha} \partial_{\alpha}$, related by equation

$t^{\alpha}=\varepsilon^{\alpha \beta} s_{\beta}, \quad\left(t^{\alpha}\right) \neq 0$,

where $\varepsilon^{\alpha \beta}:=h^{\alpha \gamma} h^{\beta \delta} \varepsilon_{\gamma \delta}$ is the totally antisymmetric second rank tensor, $\varepsilon_{12}:=\sqrt{\operatorname{det} h_{\alpha \beta}}$. They have the properties

$t^{2}-s^{2}=0, \quad(t, s)=0$,

where the scalar product is defined by $h_{\alpha \beta}$. The changing of the metric signature results in changing of one sign in the first equation in Eq. (54) as compared to Eq. (12).

After the coordinate transformation $\tau, \sigma \mapsto \tilde{\tau}, \tilde{\sigma}$ defined by Eq. (13) the metric becomes conformally flat:

$d s^{2}=h_{\alpha \beta} \frac{\partial x^{\alpha}}{\partial \tilde{x}^{\gamma}} \frac{\partial x^{\beta}}{\partial \tilde{x}^{\delta}} d \tilde{x}^{\gamma} d \tilde{x}^{\delta}=t^{2} d \tilde{\tau}^{2}+s^{2} d \tilde{\sigma}^{2}=t^{2}\left(d \tilde{\tau}+d \tilde{\sigma}^{2}\right)$,

where $t^{2}:=h_{\alpha \beta} t^{\alpha} t^{\beta}$.

Lemma 7.1 Vector fields $t$ and $s$ on $\mathbb{U}$ related by Eq. (53) commute if and only if

$t_{\alpha}=\frac{\partial_{\alpha} \chi}{\partial \chi^{2}}$

where $\chi$ is a nontrivial solution of the Laplace-Beltrami equation

$\triangle_{(h)} \chi:=h^{\alpha \beta} \nabla_{\alpha} \nabla_{\beta} \chi=0$.

For any nontrivial solution of Eq. (56) vector fields (53) and (55) commute.

Proof Repeats the proof of Lemma 4.2 which does not depend on the signature of the metric, but now we obtain the Laplace-Beltrami equation. It is well known that Eq. (56) has many solutions on a plane (harmonic functions), and there is no local extremum. Therefore the requirements $\left(\partial_{\alpha} \chi\right) \neq 0$ and $\partial \chi^{2} \neq 0$ are fulfilled automatically for any nontrivial (nonconstant) solution of Eq. (56).

So, the most general commuting vector fields having properties (54) have the same form (29) as in the Lorentzian case. The only difference is that now an arbitrary function $\chi$ satisfies the Laplace-Beltrami equation (56) instead of the wave equation (24).
To clarify the meaning of the harmonic function $\chi$ we consider the example.

Example 2 Let the initial metric be conformally flat

$d s^{2}=\mathrm{e}^{2 \phi}\left(d x^{2}+d y^{2}\right)=\mathrm{e}^{2 \phi} d z d \bar{z}$,

where $\phi(x, y)$ is a real valued function, we introduced complex coordinate $z:=x+i y$, and the bar denotes complex conjugation. After the coordinate transformation $x^{\alpha} \mapsto \tilde{x}^{\alpha}$ defined by function $\chi$ it is

$d s^{2}=t^{2}\left(d \tilde{x}^{2}+d \tilde{y}^{2}\right)=t^{2} d \tilde{z} d \bar{z}$,

where $\tilde{z}:=\tilde{x}+i \tilde{y}$ and

$t^{2}=h_{\alpha \beta} t^{\alpha} t^{\beta}=\frac{1}{\partial \chi^{2}}$.

The Laplace-Beltrami equation for conformally flat metric reduces to the Laplace equation

$\partial_{z} \partial_{\bar{z}} \chi=0$.

Its general real valued solution is

$\chi=w(z)+\bar{w}(\bar{z})$,

where $w(z)$ is an arbitrary holomorphic function. Therefore

$\partial \chi^{2}:=h^{\alpha \beta} \partial_{\alpha} \chi \partial_{\beta} \chi=\mathrm{e}^{-2 \phi} \partial_{z}(w+\bar{w}) \partial_{\bar{z}}(w+\bar{w})$

$=\mathrm{e}^{-2 \phi} \partial_{z} w \partial_{\bar{z}} \bar{w}$

and the metric is

$d s^{2}=\mathrm{e}^{2 \phi} \partial_{w} z \partial_{\bar{w}} \bar{z} d w d \bar{w}$.

Thus the transformation of coordinates defined by the harmonic function $\chi$ coincides with the conformal transformation $z \mapsto w(z)$.

The existence of vector fields $t$ and $s$ provides sufficient conditions for the existence of the conformal gauge on the whole Euclidean plane $\mathbb{R}^{2}$ for metrics separated from zero and infinity (30, and analog of Theorem 4.1 holds.

The Euclidean version of string theory is used in the path integral formulation of quantum string theory, which assumes summation over Riemannian surfaces of different genera. The Riemannian surfaces cannot be covered by a single coordinate chart, and therefore we cannot talk about the conformal gauge on the whole Riemannian surface. The results of the present section guarantee the existence of the conformal gauge on the whole coordinate chart which is diffeomorphic to $\mathbb{R}^{2}$. Previous theorems provide sufficient conditions for the existence of the conformal gauge only in some sufficiently small neighbourhood of each point of the manifold.

\section{Conclusion}

It was assumed for many years that there exists the global conformal gauge in string theory though this statement was 
proved only locally. In fact, almost all results were obtained under validity of this assumption which turns out to be true quite unexpectedly at least to the author. We proved the global existence of the conformal gauge for infinite, open, and closed strings. The transition from local to global statement is based on the global existence of the solution of the Cauchy problem for a two-dimensional hyperbolic equation with varying coefficients [7] and is far from being obvious.

As a byproduct, we proved global existence of the conformal gauge for a general two-dimensional Lorentzian metric defined on the whole plane $\mathbb{R}^{2}$ which is not necessarily induced by an embedding and is well known locally for a long time (see, e.g. $[4,5]$ ).

The existence theorem is also proved for a Riemannian positive definite metric defined on the whole Euclidean plane. It generalizes previous results providing the existence of the conformal gauge in some sufficiently small neighbourhood of each point.

Acknowledgements This work is supported by the Russian Science Foundation under grant 19-11-00320.

Data Availability Statement This manuscript has no associated data or the data will not be deposited. [Authors' comment: There are no external data associated with the manuscript.]

Open Access This article is licensed under a Creative Commons Attribution 4.0 International License, which permits use, sharing, adaptation, distribution and reproduction in any medium or format, as long as you give appropriate credit to the original author(s) and the source, provide a link to the Creative Commons licence, and indicate if changes were made. The images or other third party material in this article are included in the article's Creative Commons licence, unless indicated otherwise in a credit line to the material. If material is not included in the article's Creative Commons licence and your intended use is not permitted by statutory regulation or exceeds the permitted use, you will need to obtain permission directly from the copyright holder. To view a copy of this licence, visit http://creativecomm ons.org/licenses/by/4.0/.

Funded by SCOAP ${ }^{3}$.

\section{References}

1. M.B. Green, J.H. Schwarz, E. Witten, Superstring Theory, vol. 1, 2 (Cambridge University Press, Cambridge, 1987)

2. L. Brink, M. Henneaux, Principles of String Theory (Plenum Press, New York, 1988)

3. B.M. Barbashov, V.V. Nesterenko, Introduction to the Relativistic String Theory (World Scientific, Singapore, 1990)

4. I.G. Petrovsky, Lectures on Partial Differential Equations (Dover Publications Inc., New York, 1992)

5. V.S. Vladimirov, Equations of Mathematical Physics (Marcel Dekker, New York, 1971)

6. V.S. Vladimirov, I.V. Volovich, Boundary problems for nonlinear equations of relativistic string. Dokl. Akad. Nauk SSSR 289(5), 1043-1047 (1986) (in Russian)

7. J. Hadamard, Le Probleme de Cauchy et Les Équations Aux Dérivées Partielles Linéaires Hyperboliques (Hermann, Paris, 1932)

8. M.O. Katanaev, Global conformal gauge in string theory. Phys. Lett. B 816, 136246 (2021)

9. J.A. Wolf, Spaces of Constant Curvature (University of California, Berkley, 1972) 